\title{
Diagnostic Accuracy of Combined FP-CIT, IBZM, and MIBG Scintigraphy in the Differential Diagnosis of Degenerative Parkinsonism: A Multidimensional Statistical Approach
}

\author{
Martin Südmeyer*1,2, Christina Antke*3, Tanja Zizek ${ }^{1,2}$, Markus Beu ${ }^{3}$, Susanne Nikolaus ${ }^{3}$, Lars Wojtecki ${ }^{1,2}$, \\ Alfons Schnitzler ${ }^{1,2}$, and Hans-Wilhelm Müller ${ }^{3}$ \\ ${ }^{I}$ Department of Neurology, Faculty of Medicine and University Clinic, Heinrich Heine University, Düsseldorf, Germany; ${ }^{2}$ Institute of \\ Clinical Neuroscience and Medical Psychology, Faculty of Medicine and University Clinic, Heinrich Heine University, Düsseldorf, \\ Germany; and ${ }^{3}$ Department of Nuclear Medicine, Faculty of Medicine and University Clinic, Heinrich Heine University, Düsseldorf, \\ Germany
}

In vivo molecular imaging of pre- and postsynaptic nigrostriatal
neuronal degeneration and sympathetic cardiac innervation
with SPECT is used to distinguish idiopathic Parkinson disease
(PD) from atypical parkinsonian disorder (APD). However, the
diagnostic accuracy of these imaging approaches as stand-
alone procedures is often unsatisfying. The aim of this study
was therefore to evaluate to which extent diagnostic accuracy
can be increased by their combined use together with a
multidimensional statistical algorithm. Methods: The SPECT
radiotracers ${ }^{123}$-(S)-2-hydroxy-3-iodo-6-methoxy- $N$-[1-ethyl-2pyrrodinyl)-methyl]benzamide (IBZM), ${ }^{123}-N-\omega$-fluoropropyl$2 \beta$-carbomethoxy-3 $\beta$-(4-iodophenyl)nortropan (FP-CIT), and meta-123/-iodobenzylguanidine (MIBG) were used to assess striatal postsynaptic $D_{2}$ receptor binding, striatal presynaptic dopamine transporter binding, and myocardial adrenergic innervation, respectively. Thirty-one PD and 17 APD patients were prospectively investigated. PD and APD diagnoses were established using consensus criteria and reevaluated after $37.4 \pm 12.4$ and $26 \pm 11.6 \mathrm{mo}$ in PD and APD, respectively. Test accuracy (TA) for PD-APD differentiation was computed for all logical (Boolean) combinations of imaging modalities by receiver-operating-characteristic analysis - that is, after multidimensional optimization of cutoff values. Results: Analysis showed moderate TA for PD-APD differentiation using each molecular approach alone (IBZM, 79\%; MIBG, 73\%; and FPCIT, 73\%). For combined use, the highest TA resulted under the assumption that at least 2 of the 3 biologic markers had to be positive for APD using the following cutoff values: 1.46 or less for IBZM, less than 2.10 for FP-CIT, and greater than 1.43 for MIBG. This algorithm distinguished APD from PD with a sensitivity of $94 \%$, specificity of $94 \%$ (TA, $94 \%$ ), positive predictive value of $89 \%$, and negative predictive value of $97 \%$. Conclusion: Results suggest that the multidimensional combination of FP-CIT, IBZM, and MIBG scintigraphy is likely to significantly

Received Dec. 30, 2010; revision accepted Mar. 16, 2011. For correspondence or reprints contact either of the following:

Christina Antke, Department of Nuclear Medicine, Heinrich Heine University, University Clinic, Moorenstrasse 5, D-40225 Düsseldorf, Germany.

E-mail: antke@uni-duesseldorf.de

Martin Südmeyer, Department of Nuclear Medicine, Heinrich Heine University, University Clinic, Moorenstrasse 5, D-40225 Düsseldorf, Germany.

E-mail: martin.suedmeyer@uni-duesseldorf.de

${ }^{*}$ Contributed equally to this work.

COPYRIGHT @ 2011 by the Society of Nuclear Medicine, Inc. increase TA in differentiating PD from APD. The differential diagnosis of degenerative parkinsonism may thus be facilitated.

Key Words: Parkinson disease; atypical parkinsonian disorder; FP-CIT; IBZM; MIBG

J Nucl Med 2011; 52:733-740

DOI: 10.2967/jnumed.110.086959

$\mathbf{I}$ idiopathic Parkinson disease (PD), progressive nigrostriatal denervation and degeneration in the peripheral autonomic nervous system are typical features $(1,2)$. Clinically, this is reflected by increasing severity of characteristic motor disturbances and occurrence of axial motor symptoms such as postural abnormalities. In addition, different nonmotor symptoms may develop such as urinary or sleep disturbances (1). Although the diagnosis of PD can be unequivocal in patients with typical clinical symptoms, differentiation from atypical parkinsonian disorder (APD), such as parkinsonian type of multiple-system atrophy (MSA-P) and progressive supranuclear palsy (PSP), may be challenging in other cases (3-5). Because prognosis and treatment of patients with APD differ from those of PD patients (5), appropriate nosologic classification is warranted.

Molecular imaging techniques using PET or SPECT offer a variety of tools for diagnosing patients with parkinsonian syndromes (6-8). SPECT of the nigrostriatal dopaminergic system and of the sympathetic innervation of the heart is widely used in this context $(4,9)$. For example, imaging of dopamine transporter (DAT) binding with ${ }^{123} \mathrm{I}-\mathrm{N}$ - $\omega$-fluoropropyl$2 \beta$-carbomethoxy-3 $\beta$-(4-iodophenyl)nortropan (FP-CIT) successfully visualizes presynaptic dopaminergic degeneration of the nigrostriatal tract. This procedure allows differentiation of degenerative parkinsonism from movement disorders that are not associated with dopaminergic deficit, such as essential tremor $(4,5,10)$. DAT imaging alone, however, does not differentiate the various types of degenerative parkinsonism (PD vs. APD) with sufficient accuracy $(5,11)$. 
In the latter regard, SPECT of dopamine $\mathrm{D}_{2}$ receptors with radioligands such as ${ }^{123} \mathrm{I}-(S)$-2-hydroxy-3-iodo-6-methoxy$N$-[1-ethyl-2-pyrrodinyl)-methyl]benzamide (IBZM) may be helpful, because APD patients usually display lower $\mathrm{D}_{2}$ receptor binding than do PD patients (12-14). Similarly, scintigraphy with meta- ${ }^{123}$ I-iodobenzylguanidine (MIBG) has recently attracted interest in this context, because it also may differentiate APD and PD based on displaying pronounced cardiac sympathetic denervation in PD (15).

However, all these molecular imaging techniques are limited in terms of their test accuracy (TA). The use of FP-CIT SPECT, IBZM SPECT, or MIBG scintigraphy as stand-alone diagnostic means is therefore currently not recommended for differentiating different types of degenerative parkinsonism (11). The aim of the present study was thus to evaluate whether and to what extent the combination of FP-CIT SPECT, IBZM SPECT, and MIBG scintigraphy improved in vivo PD-APD differentiation. We here report on a first series of 48 patients with degenerative parkinsonism for whom a multidimensional statistical algorithm was applied and resulted in a marked improvement in the PDAPD discriminatory power when compared with each of the stand-alone molecular approaches.

\section{MATERIALS AND METHODS}

\section{Patients}

Between October 2005 and October 2007, a consecutive series of 48 patients with suspected degenerative parkinsonism attending the Movement Disorder Center of the University Hospital Düsseldorf was prospectively recruited. Patients underwent detailed neurologic examination (including testing of the autonomic nervous system and neuropsychologic testing), routine laboratory tests, and diagnostic brain MRI to exclude symptomatic parkinsonism, for example, multiple arteriosclerotic changes or hydrocephalus. Additionally, the Unified Parkinson Disease Rating Scale (UPDRS) (16) was determined $12 \mathrm{~h}$ after discontinuation of antiparkinsonian medication (off-state). Levodopa responsiveness was assessed by a shortterm levodopa test (oral administration of 200-250 mg levodopa) or by a clear qualitative levodopa response during clinical long-term follow-up examinations. The levodopa test was considered positive if patients revealed improvement of more than $30 \%$, compared with off-baseline.

All patients underwent molecular in vivo brain and cardiac diagnostics at initial presentation comprising presynaptic striatal DAT scintigraphy with FP-CIT, postsynaptic dopamine $\mathrm{D}_{2}$ receptor scintigraphy with IBZM, and assessment of myocardial sympathetic innervation with MIBG. Exclusion criteria were history of heart disease, including coronary artery disease, infarction, or heart failure; diabetes mellitus; polyneuropathy; dementia; current neuroleptic or tricyclic antidepressive medication; and vascular cerebral lesions.

After molecular in vivo diagnostics, all patients underwent clinical follow-up examinations for $37.4 \pm 12.4$ (PD) and $26 \pm$ 11.6 (APD) months. Final diagnosis of PD or APD was made by the consensus of 3 movement disorder specialists who were unaware of the results of molecular in vivo diagnostics, taking into consideration data of carefully assessed longitudinal neurologic follow-up examinations, possible response to dopaminergic treatment, and diagnostic MRI. Thirty-one patients were classified as having PD according to the U.K. Brain Bank criteria (17). Further, 11 patients were diagnosed as probable MSA-P according to established criteria $(18,19)$, and 6 patients fulfilled the National Institute of Neurologic Disorders and Stroke criteria for probable PSP $(20,21)$.

\section{Scintigraphic Imaging}

After the thyroid was blocked with sodium perchlorate, the tracers FP-CIT (ioflupane $\left[{ }^{123} \mathrm{I}\right]$; DaTSCAN, $184 \pm 5 \mathrm{MBq}$ ), IBZM (iolopride [ $\left.{ }^{123} \mathrm{I}\right]$; IBZM, $184 \pm 8 \mathrm{MBq}$ ), or MIBG (iobenguane $\left[{ }^{123} \mathrm{I}\right]$ injection; AdreView, $134 \pm 20 \mathrm{MBq}$ ), all from GE Healthcare, were injected intravenously as a slow bolus in a resting state. In all patients, the 3 examinations were performed within $3 \mathrm{wk}$ in randomized order, with a time interval of at least $2 \mathrm{~d}$ $(2.8 \pm 1.1 \mathrm{~d})$ between MIBG scintigraphy and subsequent FPCIT SPECT or IBZM SPECT, and an interval of, at minimum, $3 \mathrm{~d}(4.7 \pm 2.3 \mathrm{~d})$ after FP-CIT SPECT or IBZM SPECT. IBZM SPECT was performed $12 \mathrm{~h}$ after discontinuation of antiparkinsonian medication. Planar and SPECT images were acquired using a dual-head $\gamma$-camera (Prism 2000; Philips) equipped with lowenergy high-resolution collimators. The imaging data were independently analyzed by 2 raters, who were unaware of the patients' identity and clinical diagnosis.

\section{Brain Imaging: FP-CIT SPECT and IBZM SPECT}

The brain SPECT scans were acquired according to the European Association of Nuclear Medicine procedure guidelines for brain neurotransmission SPECT using ${ }^{123}$ I-labeled dopamine transporter ligands (FP-CIT) or ${ }^{123}$ I-labeled dopamine $\mathrm{D}_{2}$ receptor ligands (IBZM) (22,23). SPECT image acquisition was started $4 \mathrm{~h}$ after injection for FP-CIT and 90 min after injection for IBZM in all patients, with a $128 \times 128$ image matrix and 120 projections over $360^{\circ}$. Transversal slices were reconstructed by filtered backprojection using a low-pass filter (cutoff, 0.22 Nyquist; order, 7) and attenuation-corrected by Chang's method (24). Orbitomeatal parallel slices were created by reangulation of the dataset and 3dimensional coregistration to a normalized MRI template. Images were evaluated semiquantitatively using template-based regions of interest (ROIs) and calculation of regional binding potentials as striatum-posterior cortex (S/P) ratios (Supplemental Fig. 1A; supplemental materials are available online only at http://jnm. snmjournals.org).

\section{Cardiac Imaging: MIBG Scintigraphy}

For the assessment of myocardial MIBG uptake, planar and SPECT scans were acquired. The planar images were acquired at $4 \mathrm{~h}$ after injection ( $5 \mathrm{~min}, 128 \times 128$ matrix). Immediately before or after the acquisition of planar images, a SPECT scan of myocardial sympathetic innervation was obtained for each patient to exclude focal deficits of tracer accumulation. The SPECT images were analyzed visually after iterative reconstruction without attenuation correction. Myocardial MIBG uptake was evaluated using standardized ROIs as heart-to-mediastinum (H/M) ratio on planar images $4 \mathrm{~h}$ after injection (Supplemental Fig. 1B).

\section{Statistical Evaluation}

Group data are presented as mean \pm SD. For statistical comparison, the 2-sided $t$ test was used for normally distributed data and the 2-sided Mann-Whitney $U$ test for nonnormally distributed data. Normality of data distribution was assessed using the Kolmogorov-Smirnov test. Differences with a $P$ value of less than 
0.05 were considered biologically significant. Descriptive statistics and basic comparisons were analyzed using the Medcalc software package (version 11.1). To evaluate interrater variance, the interrater agreement statistic was computed (weighted $\kappa$ ).

One-Dimensional Analysis. Receiver-operating-characteristic (ROC) analysis to optimize threshold values in terms of sensitivity and specificity for the given imaging modality guided the interpretation of the calculated $\mathrm{S} / \mathrm{P}$ and $\mathrm{H} / \mathrm{M}$ ratios. We used $\mathrm{S} / \mathrm{P}$ ratios contralateral to the clinically most affected side for ROC analysis. Specificity, sensitivity, positive predictive value (PPV), negative predictive value (NPV), TA, area under the curve (AUC), and confidence interval (CI) were calculated for the individual imaging methods after optimization of cutoff values by ROC analysis.

Multidimensional Analysis. To test the combined use of the molecular approaches, we used logical combinations. One of the authors wrote the software. The tested combinations used to diagnose APD were if all 3 methods, or if at least 2 of the 3 methods, or as soon as just 1 method was indicative of APD.

In analogy to a 1-dimensional ROC analysis, the software calculates specificity, sensitivity, PPV, NPV, and TA for every possible "and" or "or" combination of the 3 methods and for every possible combination of cutoff value. It therefore provides the optimized combination of methods (i.e., the combination with the highest resulting TA) with the corresponding cutoff values.

\section{RESULTS}

At the time of molecular diagnostics, the mean age of PD patients $(n=31)$ was $64 \pm 8.5 \mathrm{y}$ (range, $41-74 \mathrm{y})$, and the mean age of APD patients $(n=17)$ was $65.9 \pm 8.3 \mathrm{y}$ (range, 49-78 y, $P=0.49$, Mann-Whitney $U$ test, 2 -sided). In the PD group, disease duration $(6.7 \pm 6.7 \mathrm{y})$ and disease severity (UPDRS-off score, $29.6 \pm 19.5$ ) were similar to those of the APD group (disease duration, $5.8 \pm 3.3 \mathrm{y}, P=$ 0.48 ; UPDRS-off score, $32.5 \pm 14.2, P=0.42$, MannWhitney $U$ test, 2-sided). PD patients displayed a positive response to levodopa or robust clinical improvements of motor symptoms during continuing dopaminergic therapy in all cases, whereas only 3 of $11(27 \%)$ MSA-P patients showed a levodopa responsiveness. PSP patients revealed no relevant short- or long-time levodopa response (Supplemental Table 1).

During the follow-up period, 14 PD patients (45\%) developed motor fluctuations; 8 of these patients $(26 \%)$ underwent deep brain stimulation. Three patients (10\%) died from non-PD-related disorders (gastric carcinoma, heart attack). The APD patients demonstrated a much more rapid worsening of clinical symptoms and lower long-time survival rates. All MSA-P patients $(n=11)$ developed severe urinary or fecal incontinence and hypotension, and the PSP patients $(n=6)$ demonstrated a marked postural instability with spontaneous falls and vertical supranuclear palsy. Eleven APD (9 MSA-P, 2 PSP) patients died (65\%; median survival rate, $7.81 \mathrm{y}$ ) from disease-related complications (pneumonia, suffocation, sepsis).

\section{Imaging Results}

The individual binding data are plotted in Figure 1 for all 3 molecular imaging modalities. The interrater reliability was high (for FP-CIT SPECT: weighted $\kappa, 0.972$, and sensitivity, 0.158; for IBZM SPECT: weighted $\kappa, 0.943$, and sensitivity, 0.155; and for MIBG scintigraphy: weighted $\kappa$, 0.955 , and sensitivity, 0.143).

Mean binding of FP-CIT was significantly higher in PD patients (contralateral, $1.96 \pm 0.39$; ipsilateral, $2.09 \pm 0.50$ ) than in APD patients (contralateral, $1.59 \pm 0.22$, MannWhitney $U$ test, $P=0.001$; ipsilateral, $1.68 \pm 0.32, t$ test, $P=0.004$; Fig. 1A).

Mean $\mathrm{D}_{2}$ receptor binding ratios were significantly lower in APD patients (contralateral, $1.44 \pm 0.16, t$ test; ipsilateral, $1.46 \pm 0.13, t$ test; Fig. $1 \mathrm{~B})$ than in $\mathrm{PD}$ patients (contralateral, $1.60 \pm 0.11, P=0.0007$; ipsilateral, $1.59 \pm$ $0.10, P=0.001)$.

Neither FP-CIT SPECT nor IBZM SPECT revealed significant side-to-side differences in PD or APD ( $t$ test).

Mean myocardial MIBG accumulation was significantly lower in the PD group (ratio, $1.34 \pm 0.27$ ) than in the APD group $(1.60 \pm 0.29$, Mann-Whitney $U$ test, $P=0.0039$; Fig. 1 C).

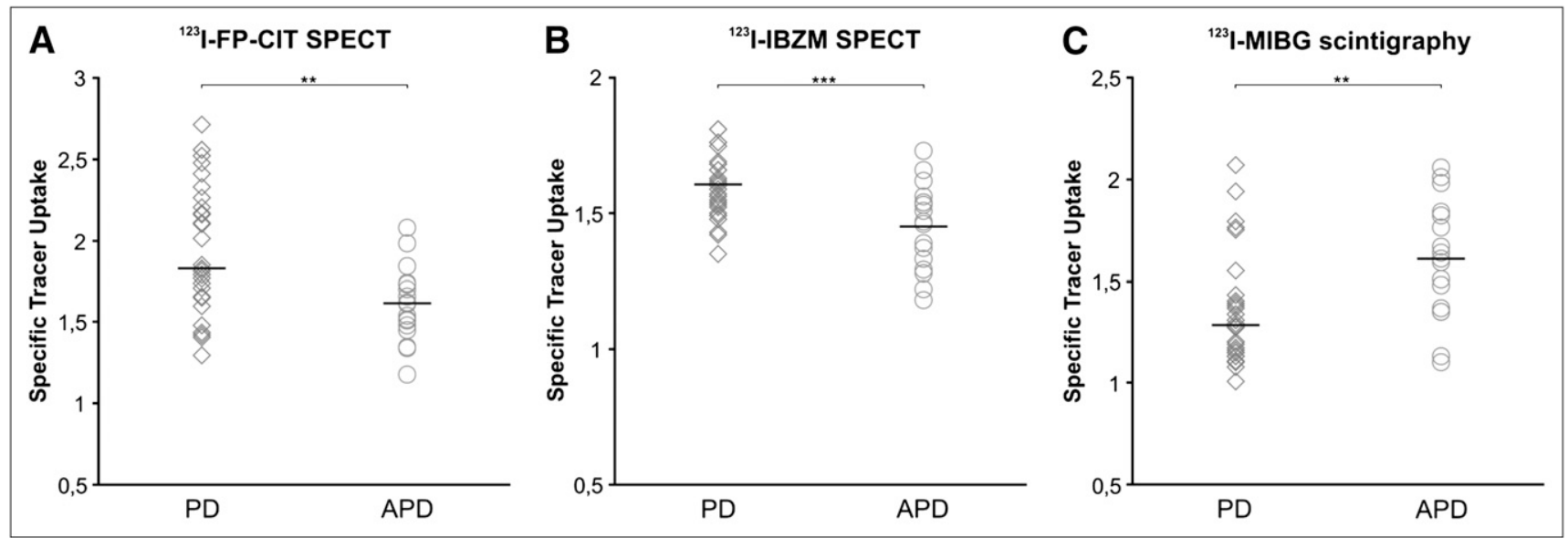

FIGURE 1. Scatterplots of striatal FP-CIT (A), striatal IBZM (B), and cardiac MIBG accumulation (C) in patients with idiopathic PD and APD. Horizontal bars indicate medians. ${ }^{* \star} P<0.01$ and ${ }^{* \star *} P<0.001$ are statistically significant group differences. 
Although statistical analysis of FP-CIT, IBZM, and MIBG values revealed significant group differences between PD and APD for each molecular imaging modality, the overlap in modalities was considerable. Thus, in individual cases, differentiation between PD and APD may be impossible, as evidenced in Figure 2, which depicts FP-CIT, IBZM, and MIBG accumulation in PD (A and C) and APD (B and D) patients. A typical combination of results is demonstrated in Figures 2A and 2B: decreased FP-CIT binding in PD and APD but maintained or even increased $\mathrm{D}_{2}$ receptor density and severely decreased cardiac MIBG accumulation in PD is shown in Figure 2A (patient 26: FP-CIT, 1.60; IBZM, 1.68; MIBG, 1.13), and maintained cardiac innervation but reduced $\mathrm{D}_{2}$ receptor binding in APD is shown in Figure 2B (patient 45: FP-CIT, 1.40; IBZM, 1.38; MIBG, 2.01).

In Figures 2C (patient 3, PD: FP-CIT, 2.33; IBZM, 1.72; MIBG, 1.75) and 2D (patient 38, APD: FP-CIT, 1.66; IBZM, 1.54; MIBG, 1.76), the results of MIBG scintigraphy and IBZM SPECT are inconclusive. In both cases, diagnoses were correctly made when considering FP-CIT SPECT results.

\section{ROC Analyses}

To identify the optimally discriminating cutoff values in the differentiation of APD and PD, we calculated ROC curves for the 3 individual imaging modalities (Fig. 3) and for all logical combinations of these molecular imaging modalities.

Individual Imaging Modalities. Using ROC analysis, we determined the following cutoff values for differentiating APD from PD: for FP-CIT SPECT, 1.73 or less (AUC, 0.775 ; 95\% CI, 0.632-0.883; Fig. 3A); for IBZM SPECT, 1.47 or less (AUC, 0.772; 95\% CI, 0.628-0.881; Fig. 3B); and for MIBG scintigraphy, more than 1.34 (AUC, 0.755; 95\% CI, 0.610-0.868; Fig. 3C). The highest TA $(79 \%)$ was evident for IBZM SPECT, with a sensitivity of $53 \%$, specificity of $94 \%$, PPV of $82 \%$, and NPV of $78 \%$. For MIBG scintigraphy, TA was $73 \%$ (sensitivity, $88 \%$; specificity, 65\%; PPV, 58\%; NPV, 91\%), and for FP-CIT SPECT, TA was 73\% (sensitivity, 76\%; specificity, 71\%; PPV, 59\%; NPV, 85\%).

Combined Imaging Modalities. We further optimized cutoff values for the possible logical combinations of the different imaging techniques. The highest TA (94\%) was reached by combining IBZM SPECT, FP-CIT SPECT, and MIBG scintigraphy under the assumption that a diagnosis of APD has to be made whenever this is suggested by the results of at least 2 of the 3 methods. Calculated cutoff values for this combination were as follows: IBZM SPECT, 1.46 or less; MIBG scintigraphy, more than 1.43; and FPCIT SPECT, less than 2.10 (Table 1, middle column). These cutoff values distinguished APD from PD with a sensitivity

FIGURE 2. FP-CIT SPECT, IBZM SPECT, and MIBG scintigraphic images for PD (A and $C)$ and $A P D(B$ and $D)$ patients. Color bars display scintillation counts. Unequivocal molecular combinations are depicted in A for PD (striatal FP-CIT decrease; myocardial MIBG decrease; normal striatal $D_{2}$ receptor binding) and in B for APD (striatal FP-CIT decrease; normal myocardial MIBG; reduced striatal $D_{2}$ receptor binding). Indecisive MIBG scintigraphy and IBZM SPECT constellations ( $C$ in PD and D in APD patients) become unequivocal after adding FP-CIT SPECT results.

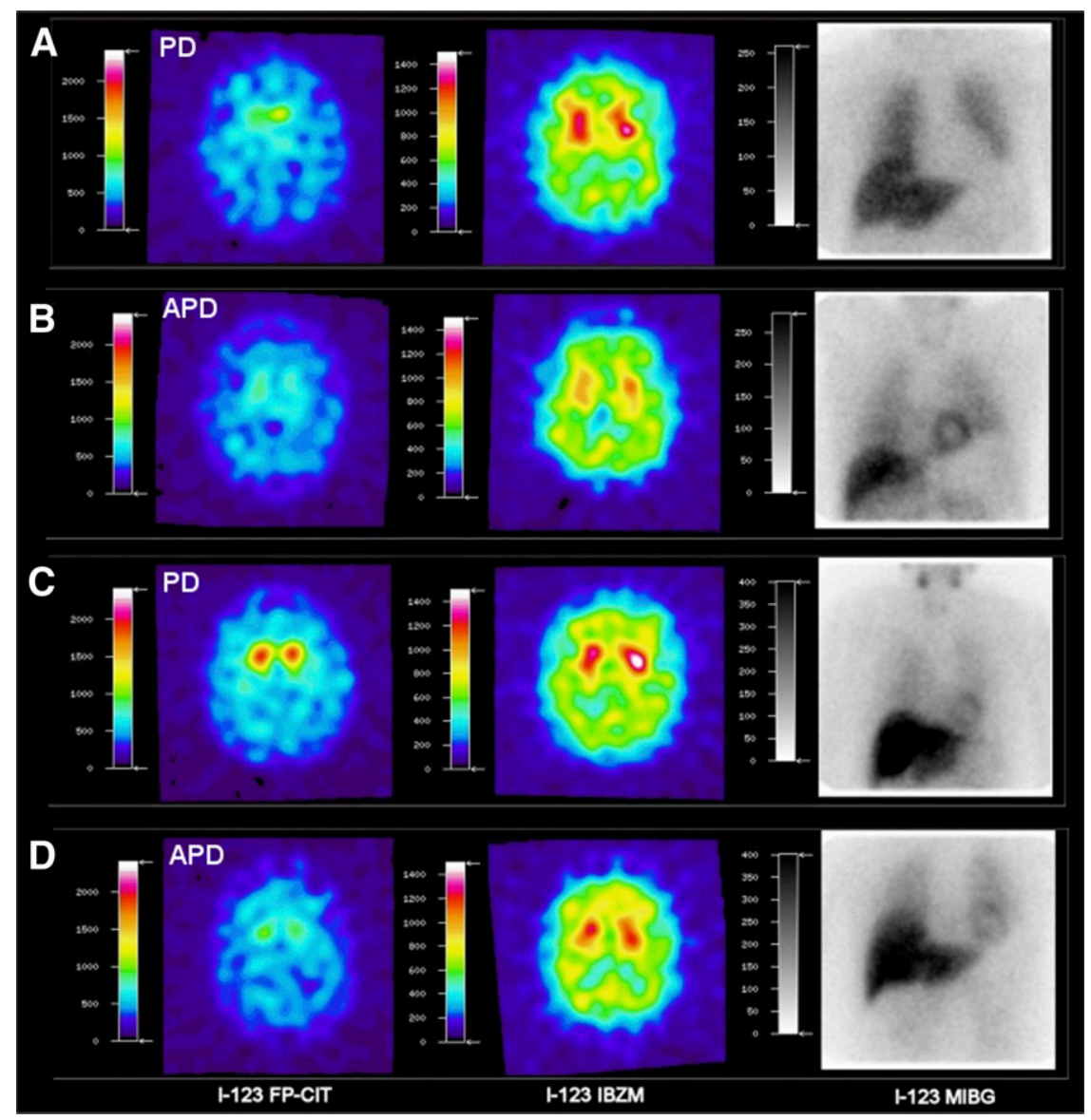




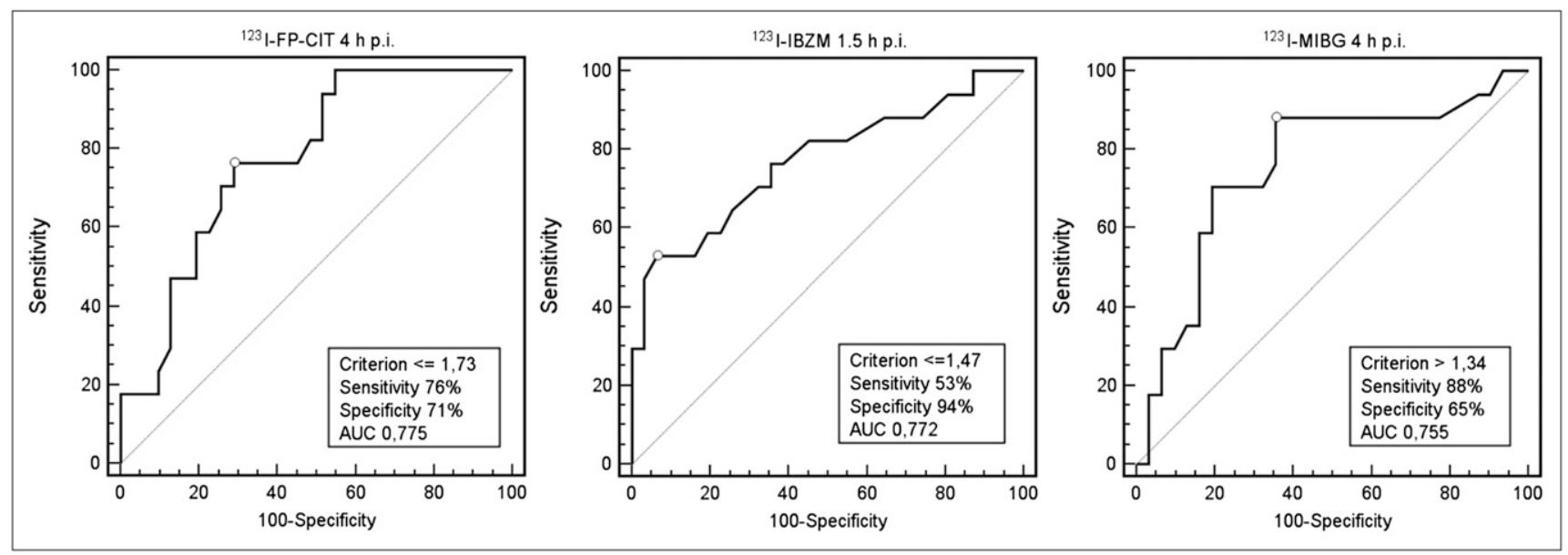

FIGURE 3. ROC curves indicating sensitivity and specificity of FP-CIT SPECT (A), IBZM SPECT (B), and MIBG scintigraphy (C) for discriminating APD from PD. Small circles on ROC curve indicate position yielding highest TA. p.i. = after injection.

of $94 \%$, a specificity of $94 \%$, a PPV of $89 \%$, and an NPV of $97 \%$.

The second best results were found for the assumption that all 3 methods must indicate APD, using the following cutoff values: IBZM SPECT, 1.73 or less; MIBG scintigraphy, 1.31 or more; and FP-CIT SPECT, less than 2.10 (Table 1, first column). Under these circumstances, TA amounts to $92 \%$, sensitivity to $88 \%$, specificity to $94 \%$, PPV to $88 \%$, and NPV to $94 \%$. The 1 -of-3 combination (Table 1, last column) reached a TA of only $83 \%$ for the APD diagnosis (sensitivity, 94\%; specificity, 77\%; PPV, $70 \%$; NPV 96\%) using the following cutoff values: IBZM SPECT, 1.46 or less; MIBG scintigraphy, 1.41 or more; and FP-CIT SPECT, 1.41 or less.

\section{Specified Results of 2-of-3 Combination}

On closer examination of the 2-of-3 combination yielding the highest TA, the results for IBZM SPECT and MIBG scintigraphy were in agreement for 29 patients (4 correctly positive, APD; 24 correctly negative, PD; and 1 falsely negative). In 19 patients, the results of IBZM SPECT and MIBG scintigraphy were discordant. Considering the results of FP-CIT SPECT in these cases led to a correctly positive result (APD) in 12 patients, a correctly negative result (PD) in 5 patients, and an incorrectly positive result in 2 patients.

\section{DISCUSSION}

This study explores to which extent the combined use of IBZM SPECT, MIBG scintigraphy, and FP-CIT SPECT and their multidimensional statistical evaluation may improve diagnostic accuracy in the differential diagnosis of degenerative parkinsonism. The analysis shows moderate TA for PD versus APD differentiation using each molecular approach alone (IBZM SPECT, 79\%; MIBG scintigraphy, 73\%; and FP-CIT SPECT, 73\%). The highest TA resulted if at least 2 of the 3 biologic markers were positive for APD

TABLE 1

Diagnosis of APD: Evaluative Statistical Parameters for Combined Use of FP-CIT SPECT, IBZM SPECT, or MIBG Scintigraphy for Differentiation of APD from PD

\begin{tabular}{cccc}
\hline Parameter & 3 of 3 & At least 2 of 3 & Any 1 of 3 \\
\hline Cutoff values & FP-CIT $<2.10$ & FP-CIT $<2.10$ & FP-CIT $\leq 1.41$ \\
& IBZM $\leq 1.73$ & IBZM $\leq 1.46$ & IBZM $\leq 1.46$ \\
Sensitivity (\%) & MIBG $\geq 1.31$ & MIBG $>1.43$ & MIBG $\geq 1.41$ \\
Specificity (\%) & 88 & 94 & 94 \\
NPV (\%) & 94 & 94 & 77 \\
PPV (\%) & 94 & 97 & 96 \\
TA (\%) & 88 & 89 & 70 \\
& 92 & 94 & 83
\end{tabular}

Cutoff values were calculated for all possible logical combinations of 3 imaging techniques and optimized using maximum TA as optimization criterion. Accordance of at least 2 of 3 biologic markers using indicated cutoff values yielded highest TA of $94 \%$ for diagnosing APD (middle column). 
using the following cutoff values: IBZM SPECT, 1.46 or less; FP-CIT SPECT, less than 2.10; and MIBG scintigraphy, more than 1.43. This algorithm distinguished APD from PD with a sensitivity of $94 \%$, a specificity of $94 \%$ (TA also $94 \%$ ), a PPV of $89 \%$, and a NPV of $97 \%$.

In clinical terms, the ability of widely available molecular imaging techniques combined with this algorithm to distinguish APD from PD with such high accuracy is remarkable, possibly allowing the correct discrimination between APD and PD to occur earlier in the disease course.

\section{Unimodal Investigations}

A series of studies has been conducted using singular molecular components of synapses as potentially facilitating the differential diagnosis of degenerative parkinsonism.

\section{Presynaptic Nigrostriatal System}

Imaging of presynaptic DAT can be assessed by a variety of SPECT and PET tracers (25). FP-CIT SPECT is thus of widespread clinical use and allows highly accurate differentiation between degenerative parkinsonism and movement disorders not associated with a dopamine deficit such as essential tremor. Plotkin et al., for example, report a sensitivity, specificity, and TA of 93\%, 100\%, and 94\%, respectively, in this context (12). In patients with degenerative parkinsonism, however, striatal DAT accumulation shows moderate TA in differentiating PD from APD, because loss of dopaminergic neurons in the substantia nigra and reduction of striatal dopamine projections are the histopathologic hallmark in all of these disorders. A sensitivity and a specificity of $81 \%$ were reported for distinguishing PD from MSA (26); these were close to our results of $76 \%$ sensitivity and $71 \%$ specificity.

Several different analyses to distinguish PD from APD based on presynaptic properties of the dopaminergic system reflect the current clinical need for differentiation. First, neuropathologic studies suggest that, compared with PD, the substantia nigra in APD is more uniformly affected in MSA and PSP, with disturbed projections to both putamen and caudate nucleus. Because in PD more ventrolateral areas of the substantia nigra and its putaminal projections are targeted, several SPECT studies have tried to objectify a specific pattern or degree of striatal DAT uptake in this area, allowing improvement of the TA of the differential diagnosis in degenerative parkinsonism (5,9,27-31). On the basis of these results, some groups have reported that in early untreated PD patients the relative sparing of the caudate nucleus in FP-CIT SPECT might be helpful in distinguishing patients with PD from patients with APD $(31,32)$, whereas others have found these results of limited value $(9,12,33)$.

Second, a few studies suggest that a left-to-right asymmetry in DAT binding may be helpful for the diagnostic characterization of PD $(28,34)$. In this context, Knudsen et al. observed that an asymmetry index of striatal DAT binding greater than $15 \%$ allowed discrimination between early-tomoderate PD and MSA, with a sensitivity of $86 \%$ (34). However, these results were obtained in a small sample and are contradictory to other findings $(9,12,31)$, including our own results, which do not reveal significant asymmetry indices in drug-treated PD patients.

Third, quantification of striatal subregions and calculation of specific DAT binding indices have not consistently been reported to distinguish different degenerative parkinsonian syndromes with high TA and therefore should be used with caution in standard clinical practice (14).

Fourth, in a study using ${ }^{123} \mathrm{I}-2 \beta$-carbomethoxy-3 $\beta$-(4iodophenyl)tropane ( $\beta$-CIT) SPECT, a highly accurate classification of patients with APD and PD has been reported for the use of an operator-independent statistical parametric mapping, which has succeeded in localizing a specific reduction of specific binding in mid brain and pons of APD patients $(30,35)$. However this voxel-based analysis technique, unlike ROI-based methods, has not yet been validated in routine clinical use and is therefore not generally recommended (4). Moreover, the results probably cannot be transferred to FP-CIT SPECT, because, compared with $\beta$-CIT, FP-CIT has a much higher affinity to the DAT and a much lower affinity to serotonin transporters, the latter being mainly responsible for accumulation of $\beta$-CIT in pons and mid brain.

Finally, the progression of DAT decline is 2 times faster in APD than in PD; therefore, longitudinal DAT imaging might become a helpful diagnostic biomarker (29).

\section{Dopamine $\mathbf{D}_{2}$ Receptors}

Upregulation of posterior putamen $\mathrm{D}_{2}$ receptor binding in early and drug-naïve degenerative parkinsonism was reported to be indicative of $\mathrm{PD}$, whereas reduction could potentially be specific for APD $(9,12)$. The use of specific striatal $\mathrm{D}_{2}$ receptor binding as a stand-alone procedure has thus been shown as a reasonable discriminating parameter in differential diagnosis of degenerative parkinsonism. Findings of a symmetrically decreased mean $\mathrm{D}_{2}$ receptor binding in APD $(9,34)$ correspond to the SPECT results of our study, but as with FP-CIT SPECT, there is a varying degree of overlap between APD and PD. For detection of APD, a TA of 70\%-90\%, a specificity of 70\%-100\%, and a sensitivity of $60 \%-87 \%$ are reported $(10,12,14,36)$. Our ROC analysis results for the isolated use of IBZM SPECT agree with these data, yielding a TA of $79 \%$, a high specificity of $94 \%$, but only a moderate sensitivity of $53 \%$.

\section{Cardiac MIBG Uptake}

For MIBG scintigraphy, the overall $\mathrm{H} / \mathrm{M}$ ratio (1.34) of the PD patients in the present study confirms previous results reporting $\mathrm{H} / \mathrm{M}$ ratios between 1.19 and 1.57 depending on disease duration, PD subtype, and (probably) also on the exact method of quantification such as size, shape, and positioning of ROIs and the use of maximum compared with mean count values (15,28,37-39). Nevertheless, in any case considerable overlap exists for the PD-APD differentiation, suggesting also that MIBG scintigraphy alone is not sufficient to distinguish these entities and that low MIBG uptake alone does not necessarily indicate PD (40). 


\section{Multimodal investigations}

In summary, the present results on the stand-alone analyses of FP-CIT SPECT, IBZM SPECT, and MIBG scintigraphy concur with previous studies reporting presynaptic nerve terminal affection, striatal $\mathrm{D}_{2}$ receptor involvement, and myocardial sympathetic dysfunction in both PD and APD patients $(9,10,12,14,31,34,41)$. Specifically, the present data reproduce the lower cardiac MIBG uptake in PD (vs. APD). Similarly, DAT and $\mathrm{D}_{2}$ receptor binding were lower in APD than in PD $(5,9,12,14,15,39)$. However, as also previously shown $(5,12$, 40), there is a substantial overlap between PD and APD for each of these 3 biologic markers that markedly limits their respective diagnostic accuracy. We therefore examined to what extent the combination of these 3 tracers would lead to an improvement of TA. Our data show that by combining FP-CIT SPECT, IBZM SPECT, and MIBG scintigraphy and using a multidimensional statistical approach, TA can be raised to $94 \%$, assuming that APD diagnosis is indicated by at least 2 of the 3 molecular approaches.

Only a few studies have evaluated the potential diagnostic benefit of combining pre- and postsynaptic dopaminergic imaging in patients with PD versus APD $(9,12,34)$. These studies reported specific binding ratios and regional differences in striatal uptake patterns for each imaging technique alone but did not develop specific cutoff values for combined pre- and postsynaptic quantitative measures. Only Koch et al. calculated the diagnostic accuracy for the combined use of IBZM SPECT and FP-CIT SPECT using an automated and observer-independent quantification method (14). They reported a higher diagnostic power for the combined use that could be further increased if a combination of pre- and postsynaptic radiotracer binding asymmetries was considered in conjunction with striatal $\mathrm{D}_{2}$ receptor binding (14). However, this approach requires a complex calculation algorithm, and sensitivity (90.3\%), specificity (73.9\%), and TA $(79 \%)$ were inferior to that of our study.

\section{Practical Considerations}

To minimize costs and radiation exposure, we suggest first combining IBZM SPECT and MIBG scintigraphy. A combination of well-preserved striatal $\mathrm{D}_{2}$ receptor binding $(\mathrm{S} / \mathrm{P}$ ratio $\geq 1.46)$ and a marked reduction of myocardial MIBG accumulation $(\mathrm{H} / \mathrm{M}$ ratio $\leq 1.43)$ are typical for $\mathrm{PD}$ and almost certainly exclude APD. On the other hand, a combination of reduced $\mathrm{D}_{2}$ receptor density (ratio $<1.46$ ) and normal or only mildly reduced cardiac sympathetic innervation, as evidenced by an $\mathrm{H} / \mathrm{M}$ ratio greater than 1.43 , is indicative of APD. In patients with borderline or contradictory results based on IBZM SPECT and MIBG scintigraphy, an additional FP-CIT SPECT scan (S/P ratio $<2.10$ ) will be helpful to confirm or exclude APD diagnosis.

Factors such as instrumentation, reconstruction, filtration, and image processing technique may influence the quantitative results of these scintigraphic measures $(22,23)$. Therefore, the ratios reported here may need adjustments according to the acquisition and processing specifics of the individual imaging facility.

\section{CONCLUSION}

Results suggest that the combination of FP-CIT SPECT, IBZM SPECT, and MIBG scintigraphy imaging may lead to a marked improvement in distinguishing PD from APD. This approach is completely based on commercially available tracers and can therefore be used in a clinical setting in the absence of short-living PET tracers. Because the present data are based on a small sample size from 1 center, confirmation of this approach in a large population is warranted using a multicenter design.

\section{DISCLOSURE STATEMENT}

The costs of publication of this article were defrayed in part by the payment of page charges. Therefore, and solely to indicate this fact, this article is hereby marked "advertisement" in accordance with 18 USC section 1734.

\section{ACKNOWLEDGMENTS}

The study was supported by the Helmholtz Research Alliance HelMA, the Stiftung für Altersforschung, and the Forschungskommission, Heinrich Heine-University, Düsseldorf (Germany).

\section{REFERENCES}

1. Poewe W. Clinical measures of progression in Parkinson's disease. Mov Disord. 2009;24(suppl 2):S671-S676.

2. Dickson DW, Braak H, Duda JE, et al. Neuropathological assessment of Parkinson's disease: refining the diagnostic criteria. Lancet Neurol. 2009;8:1150-1157.

3. Hughes AJ, Daniel SE, Ben-Shlomo Y, Lees AJ. The accuracy of diagnosis of parkinsonian syndromes in a specialist movement disorder service. Brain. 2002; 125:861-870.

4. Scherfler C, Nocker M. Dopamine transporter SPECT: how to remove subjectivity? Mov Disord. 2009;24(suppl 2):S721-S724.

5. Felicio AC, Shih MC, Godeiro-Junior C, Andrade LA, Bressan RA, Ferraz HB. Molecular imaging studies in Parkinson disease: reducing diagnostic uncertainty. Neurologist. 2009;15:6-16.

6. Nikolaus S, Antke C, Kley K, et al. Investigating the dopaminergic synapse in vivo: I. Molecular imaging studies in humans. Rev Neurosci. 2007;18:439-472.

7. Nikolaus S, Antke C, Muller HW. In vivo imaging of synaptic function in the central nervous system: I. Movement disorders and dementia. Behav Brain Res. 2009;204:1-31.

8. la Fougère $\mathrm{C}$, Pöpperl G, Levin $\mathrm{J}$, et al. The value of the dopamine $\mathrm{D} 2 / 3$ receptor ligand ${ }^{18} \mathrm{~F}$-desmethoxyfallypride for the differentiation of idiopathic and nonidiopathic parkinsonian syndromes. J Nucl Med. 2010;51:581-587.

9. Kim YJ, Ichise M, Ballinger JR, et al. Combination of dopamine transporter and D2 receptor SPECT in the diagnostic evaluation of PD, MSA, and PSP. Mov Disord. 2002;17:303-312.

10. Vlaar AM, van Kroonenburgh MJ, Kessels AG, Weber WE. Meta-analysis of the literature on diagnostic accuracy of SPECT in parkinsonian syndromes. BMC Neurol. 2007;7:27.

11. Suchowersky O, Reich S, Perlmutter J, Zesiewicz T, Gronseth G, Weiner WJ. Practice parameter: diagnosis and prognosis of new onset Parkinson disease (an evidence-based review) — report of the Quality Standards Subcommittee of the American Academy of Neurology. Neurology. 2006;66:968-975.

12. Plotkin M, Amthauer H, Klaffke S, et al. Combined ${ }^{123}$ I-FP-CIT and ${ }^{123}$ I-IBZM SPECT for the diagnosis of parkinsonian syndromes: study on 72 patients. J Neural Transm. 2005;112:677-692.

13. Vlaar AM, de Nijs T, Kessels AG, et al. Diagnostic value of ${ }^{123}$ I-ioflupane and ${ }^{123}$ I-iodobenzamide SPECT scans in 248 patients with parkinsonian syndromes. Eur Neurol. 2008;59:258-266. 
14. Koch W, Hamann C, Radau PE, Tatsch K. Does combined imaging of the preand postsynaptic dopaminergic system increase the diagnostic accuracy in the differential diagnosis of parkinsonism? Eur J Nucl Med Mol Imaging 2007;34: 1265-1273.

15. Sawada H, Oeda T, Yamamoto K, et al. Diagnostic accuracy of cardiac metaiodobenzylguanidine scintigraphy in Parkinson disease. Eur J Neurol. 2009;16: $174-182$.

16. Movement Disorder Society Task Force on Rating Scales for Parkinson's Disease. The Unified Parkinson's Disease Rating Scale (UPDRS): status and recommendations. Mov Disord. 2003;18:738-750.

17. Hughes AJ, Daniel SE, Kilford L, Lees AJ. Accuracy of clinical diagnosis of idiopathic Parkinson's disease: a clinico-pathological study of 100 cases. J Neurol Neurosurg Psychiatry. 1992;55:181-184.

18. Gilman S, Low PA, Quinn N, et al. Consensus statement on the diagnosis of multiple system atrophy. J Neurol Sci. 1999;163:94-98.

19. Gilman S, Wenning GK, Low PA, et al. Second consensus statement on the diagnosis of multiple system atrophy. Neurology. 2008;71:670-676.

20. Litvan I, Agid Y, Calne D, et al. Clinical research criteria for the diagnosis of progressive supranuclear palsy (Steele-Richardson-Olszewski syndrome): report of the NINDS-SPSP international workshop. Neurology. 1996;47:1-9.

21. Litvan I, Mangone CA, McKee A, et al. Natural history of progressive supranuclear palsy (Steele-Richardson-Olszewski syndrome) and clinical predictors of survival: a clinicopathological study. J Neurol Neurosurg Psychiatry. 1996;60: $615-620$.

22. Tatsch K, Asenbaum S, Bartenstein P, et al. European Association of Nuclear Medicine procedure guidelines for brain neurotransmission SPET using ${ }^{123} \mathrm{I}-$ labelled dopamine $\mathrm{D}_{2}$ transporter ligands. Eur J Nucl Med Mol Imaging. 2002; 29:BP30-BP35.

23. Tatsch K, Asenbaum S, Bartenstein P, et al. European Association of Nuclear Medicine procedure guidelines for brain neurotransmission SPET using ${ }^{123} \mathrm{I}-$ labelled dopamine $\mathrm{D}_{2}$ receptor ligands. Eur J Nucl Med Mol Imaging. 2002; 29:BP23-BP29.

24. Chang L. A method for attenuation correction in radionuclide computed tomography. IEEE Trans Nucl Sci 1978;NS-25:638-643.

25. Brooks DJ. Imaging dopamine transporters in Parkinson's disease. Biomarkers Med. 2010;4:651-660.

26. Varrone A, Marek KL, Jennings D, Innis RB, Seibyl JP. $\left[{ }^{123} \mathrm{I}\right] \beta-\mathrm{CIT}$ SPECT imaging demonstrates reduced density of striatal dopamine transporters in Parkinson's disease and multiple system atrophy. Mov Disord. 2001;16:1023-1032.

27. Antonini A, Benti R, De NR, et al. ${ }^{123}$ I-ioflupane/SPECT binding to striatal dopamine transporter (DAT) uptake in patients with Parkinson's disease, multiple system atrophy, and progressive supranuclear palsy. Neurol Sci. 2003;24:149-150.
28. Spiegel J, Mollers MO, Jost WH, et al. FP-CIT and MIBG scintigraphy in early Parkinson's disease. Mov Disord. 2005;20:552-561.

29. Pirker W, Djamshidian S, Asenbaum S, et al. Progression of dopaminergic degeneration in Parkinson's disease and atypical parkinsonism: a longitudinal $\beta$-CIT SPECT study. Mov Disord. 2002;17:45-53.

30. Seppi K, Scherfler C, Donnemiller E, et al. Topography of dopamine transporter availability in progressive supranuclear palsy: a voxelwise $\left[{ }^{123} \mathrm{I}\right] \beta-\mathrm{CIT}$ SPECT analysis. Arch Neurol. 2006;63:1154-1160.

31. Stoffers D, Booij J, Bosscher L, Winogrodzka A, Wolters EC, Berendse HW. Early-stage $\left[{ }^{123} \mathrm{I}\right] \beta$-CIT SPECT and long-term clinical follow-up in patients with an initial diagnosis of Parkinson's disease. Eur J Nucl Med Mol Imaging. 2005; 32:689-695.

32. Brucke T, Asenbaum S, Pirker W, et al. Measurement of the dopaminergic degeneration in Parkinson's disease with $\left[{ }^{123} \mathrm{I}\right] \beta$-CIT and SPECT: correlation with clinical findings and comparison with multiple system atrophy and progressive supranuclear palsy. J Neural Transm Suppl. 1997;50:9-24.

33. Booij J, Speelman JD, Horstink MW, Wolters EC. The clinical benefit of imaging striatal dopamine transporters with [ ${ }^{123}$ I]FP-CIT SPET in differentiating patients with presynaptic parkinsonism from those with other forms of parkinsonism. Eur J Nucl Med. 2001;28:266-272.

34. Knudsen GM, Karlsborg M, Thomsen G, et al. Imaging of dopamine transporters and D2 receptors in patients with Parkinson's disease and multiple system atrophy. Eur J Nucl Med Mol Imaging. 2004;31:1631-1638.

35. Scherfler C, Seppi K, Donnemiller E, et al. Voxel-wise analysis of $\left[{ }^{123} \mathrm{I}\right] \beta$-CIT SPECT differentiates the Parkinson variant of multiple system atrophy from idiopathic Parkinson's disease. Brain. 2005;128:1605-1612.

36. Larisch R, Klimke A. Clinical impact of cerebral dopamine-D2 receptor scintigraphy [in German]. Nuklearmedizin. 1998;37:245-250.

37. Braune $S$. The role of cardiac metaiodobenzylguanidine uptake in the differential diagnosis of parkinsonian syndromes. Clin Auton Res. 2001;11:351-355.

38. Satoh A, Serita T, Seto M, et al. Loss of ${ }^{123} \mathrm{I}-\mathrm{MIBG}$ uptake by the heart in Parkinson's disease: assessment of cardiac sympathetic denervation and diagnostic value. J Nucl Med. 1999;40:371-375.

39. Yoshita M. Differentiation of idiopathic Parkinson's disease from striatonigral degeneration and progressive supranuclear palsy using iodine-123 meta-iodobenzylguanidine myocardial scintigraphy. J Neurol Sci. 1998;155:60-67.

40. Rascol O, Schelosky L. ${ }^{123}$ I-metaiodobenzylguanidine scintigraphy in Parkinson's disease and related disorders. Mov Disord. 2009;24(suppl 2):S732S741.

41. Druschky A, Hilz MJ, Platsch G, et al. Differentiation of Parkinson's disease and multiple system atrophy in early disease stages by means of I-123-MIBGSPECT. J Neurol Sci. 2000;175:3-12. 